\title{
STUDY OF VITAMIN D LEVELS AND ITS CORRELATION WITH AMBULATORY STATUS IN STROKE SURVIVORS : A CROSS SECTIONAL STUDY.
}

Medical Science

Dr Sakshi Jain

Dr Vinay

Kanaujia

\section{Dr Dhirendra}

Kumar Singh

Dr Banoth Kiran

Kumar*

MBBS MD (PMR), Senior Resident, Department of Physical Medicine and Rehabilitation, VMMC and Safdarjung Hospital, New Delhi.

MBBS MD (PMR), Senior Resident, Department of Physical Medicine and Rehabilitation, AIIMS, Rishikesh, Uttarakhand.

MBBS MD (PMR), Senior Resident, Department of Physical Medicine and Rehabilitation, VMMC and Safdarjung Hospital, New Delhi.

MBBS DNB (PMR), Senior Resident, Department of Physical Medicine and Rehabilitation, VMMC and Safdarjung Hospital, New Delhi. ${ }^{*}$ Corresponding author

\section{ABSTRACT}

Background - Stroke can lead to impaired mobility and disability. There are chances of decreased exposure to sunlight and decreased Vitamin D levels after stroke. This study was done to assess the vitamin D status in stroke survivors and its correlation with duration and ambulatory status of stroke.

Methods - This cross sectional study included 100 patients of stroke. Patients were assessed after history and examination for their Serum Vitamin D levels, ambulatory status by Modified Rankin Scale (MRS). MRS grade 1-3 were considered ambulatory and MRS grade 4 and 5 were considered non ambulatory. Statistical analysis was done using chi square test for qualitative variables, unpaired test for quantitative variables, and pearson correlation. P value $<0.05$ was taken significant.

Results -72 patients had Vitamin D levels less than 30ng/ml $(20.42 \pm 5.89 \mathrm{ng} / \mathrm{ml})$. Significant negative correlation was observed between duration of stroke and Serum Vitamin D levels. Out of 41 non ambulatory patients, 38 were Vitamin D deficient $(\mathrm{P}<0.05)$ Negative. Correlation was present in Modified Rankin Scale and Serum Vitamin D levels.

Conclusion -Vitamin D Deficiency is present in chronic non ambulatory stroke patients.

\section{KEYWORDS}

Stroke, Vitamin D, Vitamin D deficiency, Modified Rankin Scale

\section{INTRODUCTION}

Stroke is defined as "rapidly developed clinical signs of focal (or global) disturbance of cerebral function, lasting more than 24 hours or leading to death, with no apparent cause other than of vascular origin". Stroke is one of the major causes of disability and death. In India, the estimated adjusted prevalence rate of stroke ranges from 84$262 / 100,000$ in rural areas and $334-424 / 100,000$ in urban areas. Its incidence rate is $119-145 / 100,000$ based on the population based studies.

Stroke can lead to motor impairment, sensory disturbances, visual impairment, movement problems, dysphagia, aphasia, bladder and bowel problems, cognitive impairments and emotional disturbances. ${ }^{3}$ These motor and cognitive deficits can lead to impaired mobility and decreased bone load in stroke survivors. This impaired mobility in outdoors can lead to less exposure to sunlight. Also, stroke patients get poor nutrition due to various reasons such as age, mobility impairment, dysphagia, cognitive deficits. ${ }^{4}$ As source of Vitamin D is sunlight exposure and dietary supplements, ${ }^{5}$ stroke survivors can be Vitamin D deficient due to impaired mobility, motor deficits, cognitive deficits and swallowing difficulties. Thus, assessment of Vitamin D levels after stroke can prevent Vitamin D Deficiency in stroke survivors and may prevent bone loss and risk of fractures in those patients.

This study is aimed to assess changes in Vitamin D levels in stroke survivors and also its correlation with duration of stroke and ambulatory status of patients.

\section{MATERIALS AND METHODS}

This single centered cross-sectional observational study was conducted in the department of Physical Medicine and Rehabilitation of a tertiary care hospital over the duration of six months from September 2018 to March 2019. Permission for record-based study was taken from Institutional Review Board. Subjects who fulfilled the inclusion criteria (Diagnosed cases of stroke on Computed Tomography Scan with age more than 18 years) and exclusion criteria (Concomitant fracture, bone malignancy, metabolic bone disease,premorbid mobility, chronic liver disease, renal disease, intestinal malabsorption, patients who are on drugs which have effect on bone metabolism like bisphosphonates and steroid, patients taking vitamin D supplements within last 6 months) were enrolled in the study after obtaining informed written consent.

Patients were assessed for detailed medical history, demographic characteristics and thorough clinical examination and data was recorded in pre-structured proforma. The severity of stroke was assessed using modified Rankin Scale (MRS). ${ }^{6}$ Patients with MRS Grade 1,2 and 3 were considered as ambulatory and patients with MRS Grade 4 and 5 were considered non ambulatory. Serum 25-OH-vitamin D levels, measured by enzyme-linked immunosorbent assay (ELISA), were noted in all the patients. It has been accepted that if the level of serum $25(\mathrm{OH}) \mathrm{D}$ is $>30 \mathrm{ng} / \mathrm{mL}$, the level of Vitamin D is adequate and if it is $<30 \mathrm{ng} / \mathrm{mL}$, there is Vitamin D insufficiency or deficiency. Patients were divided into two groups as Vitamin D deficient (Group 1) with a Vitamin D level below $30 \mathrm{ng} / \mathrm{mL}$ and normal (Group 2) with a value above $30 \mathrm{ng} / \mathrm{mL}$

\section{Statistical Analysis:}

Data was collected and entered in MS exceland was analyzed usingStatistical Package for Social Sciences (SPSS) version 21.0. Categorical variables were presented as number and percentage and numerical variables as mean $\pm \mathrm{SD}$. Normality of data was tested by Kolmogorov-Smirnov test.Chi square test and unpaired t test was used to test statistical difference between qualitative variables and quantitative variables respectively between two groups. Spearman correlation coefficient was used to test correlation between duration of stroke and Vitamin D levels and also between MRS grading and Vitamin D levels.

\section{RESULTS-}

100 patients were enrolled in study. The mean levels of serum Vitamin D was $24.67 \pm 8.77 \mathrm{ng} / \mathrm{ml} .72$ patients were in vitamin D deficient group having mean levels of serum vitamin D $20.42 \pm 5.89 \mathrm{ng} / \mathrm{ml}$ while 28 patients were in normal Vitamin D group with mean levels of $35.67 \pm 4.09 \mathrm{ng} / \mathrm{ml}$

The mean age of patients enrolled was $47.22 \pm 11.35$ years. In vitamin D deficient group the mean age was $48.33 \pm 11.30$ years while in Vitamin D normal group it was $44.36 \pm 10.80$ years. Males outnumbered females in both the groups. There were 55 males and 17 females in vitamin D deficient group whereas 20 males and 8 females were having normal Vitamin D levels.48 patients were having hemorrhagic type of 
stroke out of which 31 were Vitamin D deficient while 52 patients were having ischemic type of stroke out of which 41 were Vitamin D deficient. 37 patients were left hemiparetic and vitamin D deficient whereas 18 left hemiparetic patients were having normal Vitamin D levels. All these baseline characteristics were having insignificant difference on comparison between both the groups. (Table 1)

Table 1 - Comparison of demographic characteristics of patients between two groups

\begin{tabular}{|c|c|c|c|c|}
\hline \multicolumn{2}{|c|}{ Characteristics } & \begin{tabular}{|c} 
Vit D Deficient \\
Group \\
(Vit $\mathbf{D}<\mathbf{3 0}$ \\
ng/ml) \\
$\mathbf{N}=\mathbf{7 2}$ \\
\end{tabular} & \begin{tabular}{|c|} 
Vit D Normal \\
Group \\
(Vit $\mathrm{D} \geq \mathbf{3 0}$ \\
$\mathbf{n g} / \mathbf{m l})$ \\
$\mathbf{N}=\mathbf{2 8}$ \\
\end{tabular} & P value \\
\hline \multicolumn{2}{|c|}{ Vitamin D (ng/ml) } & $20.42 \pm 5.89$ & $35.67 \pm 4.09$ & \\
\hline Age (years) & & $48.33 \pm 11.30$ & $44.36 \pm 10.80$ & 0.113 \\
\hline \multirow[t]{2}{*}{ Gender } & Male & 55 & 20 & \multirow[t]{2}{*}{0.61} \\
\hline & Female & 17 & 8 & \\
\hline \multirow{2}{*}{$\begin{array}{l}\text { Type of } \\
\text { stroke }\end{array}$} & Hemorrhagic & 31 & 17 & \multirow[t]{2}{*}{0.112} \\
\hline & Ischemic & 41 & 11 & \\
\hline \multirow{2}{*}{\begin{tabular}{c|} 
Side Of \\
Hemiparesis
\end{tabular}} & Right & 35 & 10 & \multirow[t]{2}{*}{0.244} \\
\hline & Left & 37 & 18 & \\
\hline \multicolumn{2}{|c|}{ Duration (months) } & $7.54 \pm 2.96$ & $3.5 \pm 1.91$ & \multirow[t]{2}{*}{$<0.0001$} \\
\hline MRS & $3.58 \pm 0.96$ & $2.53 \pm 0.88$ & $<0.0001$ & \\
\hline \multirow{2}{*}{$\begin{array}{c}\text { Ambulatory } \\
\text { Status }\end{array}$} & Ambulatory & 34 & 25 & \multirow[t]{2}{*}{$<0.0001$} \\
\hline & $\begin{array}{c}\text { Non } \\
\text { Ambulatory }\end{array}$ & 38 & 3 & \\
\hline
\end{tabular}

The mean duration of stroke in patients was $6.41 \pm 3.26$ months. Patients who were having Vitamin D levels less than $30 \mathrm{ng} / \mathrm{ml}$ were having stroke from $7.54 \pm 2.96$ months while those having normal vitamin D levels were affected from stroke with mean duration of $3.5 \pm$ 1.91 months. This difference was statistical significant $(\mathrm{p}<0.0001)$ (Table 1). Significant correlation was found between duration of stroke and vitamin $\mathrm{D}$ levels on evaluation with spearman correlation $(\mathrm{p}<0.0001)$. (Figure 1)

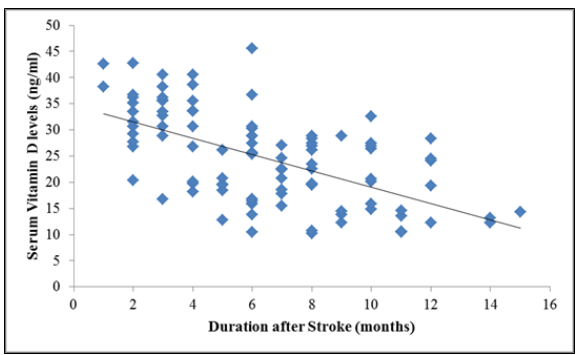

Figure 1 - Scatter diagram showing correlation between Duration after Stroke and Serum Vitamin D levels

59 patients were ambulatory and 41 were non ambulatory. Out of 51 ambulatory patients 34 were Vitamin D deficient whereas 25 patients had normal vitamin D levels. Among 41 non ambulatory patients, only 3 patients were having normal Vitamin D levels, rest were Vitamin D deficient. This was statistical significant on comparison with chi square test (Table 1). The mean MRS was 3.29 1.05. Among Vitamin D deficient patients mean MRS score was $3.58 \pm 0.96$ while it was less in patients having normal Vitamin D levels with mean MRS score of $2.53 \pm 0.88$. This difference was significant statistically $(p<0.05)$. Significant negative correlation was found between MRS score and Vitamin D levels $(p<0.0001)$. (Figure 2)

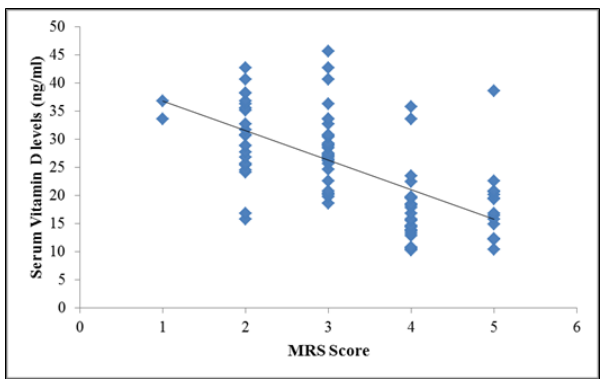

Figure 2 - Scatter diagram showing correlation between MRS score and Serum Vitamin D levels
DISCUSSION:-

In the recent years advancements in the management of stroke has increased the survival rate. In stroke survivor there are some preventable factors which can limit the disability and ultimately improve the quality of life. Vitamin D assessment is one of them and it is simple, non invasive and easily correctable.

In this study, the mean age of patients enrolled was $47.22 \pm 11.35$ years. In Stroke epidemiogical study in India, mean age of onset of stroke was found to be 54.5 years in Bangalore registry. But Mumbai and Trivandrum registries showed that the mean age of patients with stroke was 66 and 67 years respectively. ${ }^{2}$ In present study, males were more as compared to females. Similarly, higher incidence of stroke was observed in males as compared to females in Mumbai and Bangalore registry of stroke survey.

The mean duration from onset of stroke was $6.41 \pm 3.26$ months in the study. However, among vitamin D deficient patients mean duration was more than that of patients having normal vitamin D levels. Vitamin D levels were negatively correlated with duration of stroke. Similar findings were reported by Kim et al. in a cross sectional study of fifty one stroke patients. They found decrement of Vitamin D levels early after stroke onset and vitamin d levels were lower in subacute patients as compared to chronic patients. ${ }^{7}$ This suggests decrease synthesis of vitamin $\mathrm{D}$ after stroke may be due to decreased exposure to sunlight or inadequate diet after stroke.

The mean MRS levels in the present study was found to be $3.58 \pm 0.96$ in vitamin $\mathrm{D}$ deficient patients and it was lower (2.53 \pm 0.88$)$ among patients having normal Vitamin D levels. Also large number of vitamin D deficient patients was non ambulatory while maximum patients with normal vitamin D levels were ambulatory. This correlation among Vitamin D levels and MRS score and ambulatory status of patients were significant. These findings were in concordance with previous studies. ${ }^{7.8}$ Alfieri et al. conducted a prospective observational study on168 acute ischemic stroke patients to evaluate the association of vitamin D levels with acute ischemic stroke and its short-term outcome. They found that patients with poor outcome (MRS - 3-5) presented with lower vitamin D levels than those with good outcome (MRS- 0-2) after 3 months of onset of stroke. Moreover, Vitamin D Levels were negatively correlated with MRS scores after 3 months follow-up. ${ }^{8}$ Similarly, in a study done in Korea, researchers found that in terms of long-term care of stroke patients, non-ambulatory patients might be at a higher risk of vitamin D deficiency. Correlations between immobility and decrement of serum vitamin D in stroke patients were also identified by them. Patients capable of independent walking had higher levels of vitamin D than non-ambulatory patients in the chronic group. This may be due to reason that decreased mobility restricts outdoor activity and decreased exposure to sunlight in stroke patients which leadsto decreased synthesis of vitamin D.

Decreased Vitamin D levels causes secondary hyperparathyroidism. But in stroke patients, immobilization can cause hypercalcemia which suppresses release of parathyroid hormone and further decrease vitamin D3 synthesis in kidney. ${ }^{9}$ These decreased Vitamin d levels in stroke patients can lead to osteopenia and increased risk of fractures.

From this study it is concluded that chronic non ambulatory Stroke patients have prevalent Vitamin D deficiency. Therefore it is recommended to screen all stroke patients for serum vitamin $\mathrm{D}$ levels and if deficient, treatment to be started in early phase to prevent osteopenia and decrease risk of fractures.

Though there are limitations to this study. It was cross sectional observational study and results were neither compared to controls nor compared with further follow-up of patients. This study was conducted with relatively small sample size. Further studies with larger sample size and controlled trials are recommended to correlate these findings.

\section{REFERENCES:-}

1. Aho K, Harmsen P, Hatano S, Marquardsen J, Smirnov VE, Strasser T. Cerebrovascular disease in the community: results of a WHO collaborative study. Bull World Health Organ. 1980;58(1):113-30

2. Pandian JD, Sudhan P. Stroke epidemiology and stroke care services in India. J Stroke. 2013 Sep; 15(3):128-34.

3. Lui SK, Nguyen MH. Elderly Stroke Rehabilitation: Overcoming the Complication and Its Associated Challenges. CurrGerontolGeriatr Res. 2018 Jun 27:2018:9853837. Bouz SD, Tziomas K. Malnutriton in patients with acute stroke. J Nutr 2011:2011:167898.

Glerup H, Mikkelsen K, Poulsen L, Hass E, Overbeck S, Thomsen J, Charles P, Eriksen $\mathrm{EF}$. Commonly recommended daily intake of vitamin D is not sufficient if sunlight 
exposure is limited. J Intern Med. $2000 \mathrm{Feb} ; 247(2): 260-8$

vanSwieten JC, Koudstaal PJ, Visser MC, Schouten HJ, van Gijn J. Interobserver agreement for the assessment of handicap in stroke patients. Stroke. 1988 May; 19(5):604-7.

7. Kim K, Cho KH, Im SH, Choi J, Yu J, Kim M. Decrement of Serum Vitamin D Level After Stroke. Ann Rehabil Med. 2017 Dec;41(6):944-50.

8. Alfieri DF, Lehmann MF, Oliveira SR, Flauzino T, Delongui F, de Araújo MC, Dichi I, Delfino VD, Mezzaroba L, Simão AN, Reiche EM. Vitamin D deficiency is associated with acute ischemic stroke, C-reactive protein, and short-term outcome. Metab Brain Dis. 2017 Apr;32(2):493-502.

9. Sato Y, Oizumi K, Kuno H, Kaji M: Effect of immobilization upon renal synthesis of 1,25- dihydroxyvitamin D in disabled elderly stroke patients. Bone 1999;24:271-275. 